\title{
Restauration de mangroves : développement d'une solution innovante et basée sur la nature (projet ROOT)
}

\section{Mathis COGNAT ${ }^{1}$, Julien DALLE ${ }^{1}$, Alice GAVOILLE ${ }^{1}$, Alexis BEUDIN ${ }^{2}$, Benoit WAELES ${ }^{2}$, Michel BENOIT ${ }^{3}$, Philippe BLANC ${ }^{4}$}

1. SEABOOST, 889 rue de la Vieille Poste 34000 Montpellier, France. julien.dalle@seaboost.fr ; mathis.cognat@seaboost.fr

2. BW-CGC, 3 rue du commandant Groix, 29200 Brest, France.

alexis.beudin@bw-cgc.fr ; benoit.waeles@bw-cgc.fr

3. Aix Marseille Univ., CNRS, Centrale Marseille, Irphé (UMR 7342), Marseille, France. benoit@irphe.univ-mrs.fr

4. TOTAL SA, CSTJF, Av Larribau, 64018 PAU, France.

philippe.blanc@total.com

\section{Résumé :}

Dans les régions tropicales équatoriales et subtropicales, les forêts de palétuviers, ou mangroves, sont des habitats productifs qui apportent de nombreux services dont dépendent non seulement la biodiversité locale mais aussi les populations environnantes. Les palétuviers sont des espèces ingénieures de l'écosystème, ils dissipent une partie de l'énergie des vagues et ralentissent localement les courants grâce à leur système racinaire. Ils constituent ainsi des protections côtières naturelles.

Dans le contexte de régression globale des mangroves (-30\% de recouvrement sur les trois dernières décennies), des programmes de replantation de palétuviers sont conduits. Les programmes mis en œuvre jusqu'ici ont montré des taux de survie des transplants généralement faibles pour plusieurs raisons: insertion ou réinsertion d'espèces inappropriées ou implantation dans des contextes hydrosédimentaires qui ont évolué et ne leur sont plus favorables par exemple.

Issu de ce constat, le projet ROOT vise à concevoir une méthode de restauration de zones de mangroves dégradées par la réhabilitation des conditions hydrosédimentaires favorables à la recolonisation spontanée et à la croissance des palétuviers. Pour ce faire, la démarche repose sur le déploiement de modules innovants biomimétiques des racines échasses et des pneumatophores afin de reproduire l'atténuation des vagues ainsi que la rétention et le dépôt sédimentaire caractéristiques des environnements de mangrove.

Mots-clés :

Restauration mangrove, Contexte hydrosédimentaire, Biomimétisme, Modèle numérique, Modèle physique. 


\section{Thème 6 - Gestion durable des zones littorales et estuariennes}

\section{Introduction}

Dans les régions tropicales, équatoriales et subtropicales, les forêts de palétuviers, ou mangroves, sont des habitats productifs qui supportent de nombreuses fonctionnalités dont dépendent non seulement la biodiversité locale (refuge, habitat, nourricerie, frayère, ...) mais aussi les populations environnantes au-travers des services dérivés (production halieutique, matière première, protection côtière ...). Par leurs systèmes racinaires complexes, les palétuviers modifient les conditions physico-chimiques du milieu. Ils vont, par exemple, participer à l'oxygénation du substrat ou encore à la création d'un environnement de faible énergie en dissipant une partie de l'énergie des vagues incidente et en ralentissant les courants (MAZDA et al., 1997 ; NARAYAN et al., 2016). Cette capacité à modifier les facteurs biotiques et abiotiques leur confère le rôle d'ingénieur de l'écosystème (JONES et al., 1994).

Ces espèces sont reconnues pour leur action sur la dynamique sédimentaire : ils réduisent la remise en suspension des sédiments et favorisent leur dépôt (ZHANG et al., 1996 ; FAGHERAZZI et al., 2017). Ces différents effets contribuent à la stabilité du substrat, élément essentiel à la pérennité de la mangrove. Ainsi, les interactions physiques entre les palétuviers et le milieu créent des boucles de rétrocontrôle positives qui engendre des états stables alternatifs (SCHEFFER et al., 2001) : la présence des palétuviers catalyse l'installation d'autres palétuviers et donc la progression de la mangrove, et inversement en cas de perturbation / dégradation de ces espèces.

Avec l'intensification des effets du changement climatique et des activités humaines, les palétuviers jouent un rôle essentiel pour les communautés locales puisqu'ils sont des protections côtières naturelles contre l'érosion et les submersion marines.

Les mangroves sont cependant soumises à une régression alarmante. Dans ce contexte, les programmes de replantation des palétuviers ont été mis en œuvre durant les dernières décennies (SAENGER \& SIDDIQI, 1993 ; SCHMITT, 2013) mais les taux de survie des transplants sont généralement faibles pour plusieurs raisons: l'inadéquation des méthodes de plantation, l'insertion ou la réinsertion d'espèces inappropriées ou encore leur implantation dans des contextes hydrosédimentaires qui ont évolué et ne leur sont plus favorables (LEWIS, 2005 ; MARCHAND, 2008).

Issu de ce constat, le projet ROOT vise à concevoir une méthode de restauration d'un contexte hydrosédimentaire favorable à la recolonisation spontanée de zones dégradées par les palétuviers. Ces écosystèmes sont extrêmement productifs lorsque les conditions physico chimiques le permettent, il est donc raisonnable de fonder une technique de restauration basée sur leur capacité de reproduction et de dissémination élevée en créant des conditions adaptées à leur implantation. La démarche repose sur le déploiement de modules innovants biomimétiques des racines échasses (Rhizophora par exemple) et des pneumatophores (Sonneratia alba ou Avicenia par exemple) afin de reproduire d'une part l'atténuation des vagues, et d'autre part, le dépôt sédimentaire et la rétention de propagules caractéristiques des environnements de mangrove. 


\section{XVI'èmes Journées Nationales Génie Côtier - Génie Civil \\ Le Havre 2020}

Le projet ROOT est composé de 3 chantiers principaux :

- Le chantier ROOT Recherche a vocation à déterminer l'ensemble des propriétés (structure, constituants, organisation spatiale, modèle de production et de déploiement, ...) de la solution ROOT de manière à ce qu'elle remplisse ses fonctions d'atténuation de houle, de fixation du substrat et de restauration écologique.

- Le chantier ROOT Développement doit définir précisément ses caractéristiques et finaliser la conception de la solution et de son mode opératoire. Il a également pour objectif d'établir l'approche sociétale visant à créer localement des bénéfices écosystémiques à destination des communautés littorales, et ce avec leur participation. Enfin, ce chantier vise la construction d'un modèle numérique de simulation du schéma de déploiement de ROOT dans un contexte géographique donné au titre d'outil d'aide à la décision et à la mise en œuvre opérationnelle.

- Le chantier ROOT Démonstrateur doit permettre la mise en œuvre d'un démonstrateur de la solution ROOT sur un site présélectionné pour valider l'efficacité de la solution et mettre en lumière les ajustements nécessaires.

\section{Description du mode d'action de ROOT}

La solution ROOT est une solution de restauration écologique destinée aux environnements de mangrove. Elle a vocation à être déployée sur des sites sur lesquels la mangrove a été dégradée (pollution, événements climatiques, déforestation, ...) et où elle ne parvient pas, ou mal, à se restaurer de façon autonome à cause de conditions environnementales qui ne lui sont plus favorables. Il est entendu que cette technique a vocation à être appliquée dans des environnements où les pressions à l'origine des dégradations initiales sont maîtrisées et permettent d'envisager une action de restauration. Plusieurs études ont montré l'importance et la prépondérance de critères physiques et chimiques dans le développement et la pérennité d'une mangrove, tels que par exemple la topographie, les conditions hydrodynamiques et sédimentaires ou encore le niveau de salinité (LEWIS, 2005). Partant du multiple constat que :

- les projets de restauration de mangrove reposent en grande majorité sur des actions de replantation de palétuviers, sans actions (ou partielles) préalable sur les critères précités, et que ces projets aboutissent à des taux de survie faibles (BLASCO et al., 2001),

- l'efficacité de nouvelles solutions dites "douces" commencent à être prouvée (e.g., VAN CUONG et al., 2015) et que les solutions de restauration traditionnelles (briselames, épis, jetées, ...) basés sur la protection du trait de côte sont inadéquates (BULLERI \& CHAPMAN, 2010),

- la mangrove est un écosystème extrêmement productif capable de progresser latéralement de plusieurs mètres à plusieurs centaines de mètres par an selon les zones géographiques et les espèces (CLOUGH, 1992), 


\section{Thème 6 - Gestion durable des zones littorales et estuariennes}

- le succès de l'établissement de propagules sur un substrat sédimentaire nu et donc du développement de palétuviers pionniers nécessite une fenêtre d'opportunité (i.e. BALKE, 2011) assez large afin de permettre au plant de résister au contraintes hydrodynamiques par le développement suffisant des racines qui constituent l'ancrage dans le substrat,

nous avons choisi de développer une approche de restauration qui vise le rétablissement pérenne de conditions environnementales physiques propices à la réinstallation spontanée de la mangrove, à partir des mangroves adjacentes et voisines.

La réussite d'une telle solution passe par la maitrise du forçage hydrodynamique de la zone de restauration pour, d'une part, favoriser l'ancrage de propagules et d'autre part, préserver ou rétablir les caractéristiques du substrat favorable au développement et à la survie de jeunes palétuviers. Il s'agit finalement de reconstituer l'environnement énergétiquement faible dans les zones de recolonisation potentielles tout en favorisant des veines d'écoulement afin de permettre la conservation des flux hydrodynamiques. En effet, dans un contexte hydrosédimentaire approprié, un palétuvier met seulement quelques heures à s'ancrer spontanément dans le substrat grâce au développement éclair d'une racine pivot.

La figure 1 illustre le principe d'action et de déploiement de la solution ROOT. Lors d'une forte perturbation des conditions environnementales (contamination, tempête, canicule, forte variation de salinité, ...), la diminution de l'emprise spatiale d'une mangrove saine (figure 1a.) conduit à une dégradation des conditions hydrosédimentaires du milieu puisque les palétuviers ne sont plus présents pour jouer leurs rôles de rétention sédimentaire et d'atténuation des vagues et des courants. Les conditions physiques ne sont alors plus propices à la recolonisation spontanée malgré une perturbation qui n'était que temporaire (figure 1b.). C'est dans un tel contexte que la solution ROOT est vouée à être déployée (figure 1c.). Elle a pour but de maintenir les fonctions physiques (dissipation de l'énergie des vagues et des courants, rétention des propagules) des palétuviers disparus afin de favoriser la germination et le développement de jeunes palétuviers (figure 1d.), qui, une fois bien développés, permettront le retour d'un écosystème de mangrove sain et diversifié et donc de la globalité des fonctions écologiques associées (figure 1e). 


\section{XVİ̀mes Journées Nationales Génie Côtier - Génie Civil \\ Le Havre 2020}

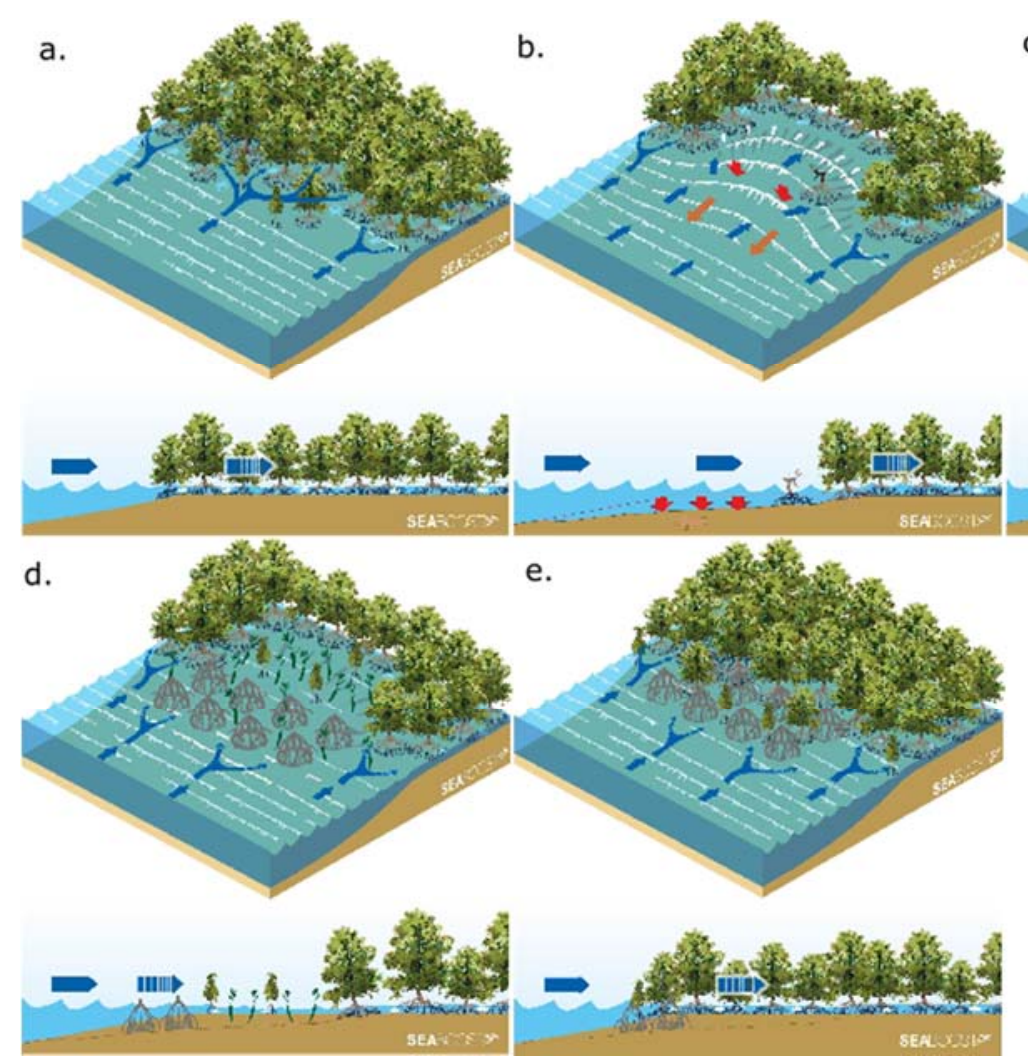

Figure 1. Principe d'action et de déploiement de la solution ROOT : a. Mangrove saine avant perturbation ; $b$. Mangrove dégradée, vulnérable aux fortes houles et à l'érosion ; c. Déploiement de la solution ROOT pour restaurer des conditions hydrosédimentaires ; $d$. La solution ROOT favorise la régénération des palétuviers et $e$. Intégration complète de la solution ROOT dans la forêt de mangrove restaurée.

Bien que cette solution vise principalement à restaurer les conditions physiques du milieu, elle pourra également porter transitoirement, du fait de ses caractéristiques structurelles, les fonctionnalités d'habitat et de nurserie pour les espèces locales le temps que la mangrove naturelle se régénère. Elle permettra ainsi de manière presque immédiate à son déploiement la restauration ou le renforcement des écosystèmes inféodés aux zones de mangroves, et la génération de ressources (halieutique, conchylicole, ...) au bénéfice des communautés locales. Idéalement, ces mêmes acteurs acquerront une autonomie totale dans la gestion, la maintenance et l'exploitation des dispositifs et seront formés à cette fin.

\section{Résultats des premiers travaux}

La première phase du projet-ROOT Recherche-a permis d'établir certaines corrélations entre des propriétés structurelles des réseaux racinaires des palétuviers et leur performance hydrodynamique, de manière à guider les chantiers de conception et de dimensionnement des objets. Trois types de tests ont permis de tester plusieurs propriétés 


\section{Thème 6 - Gestion durable des zones littorales et estuariennes}

face à différentes configurations de houle : 2 types de tests en modèles physiques (figure 2), i.e. en hexapode et en canal à houle, et des tests en modèles numériques.

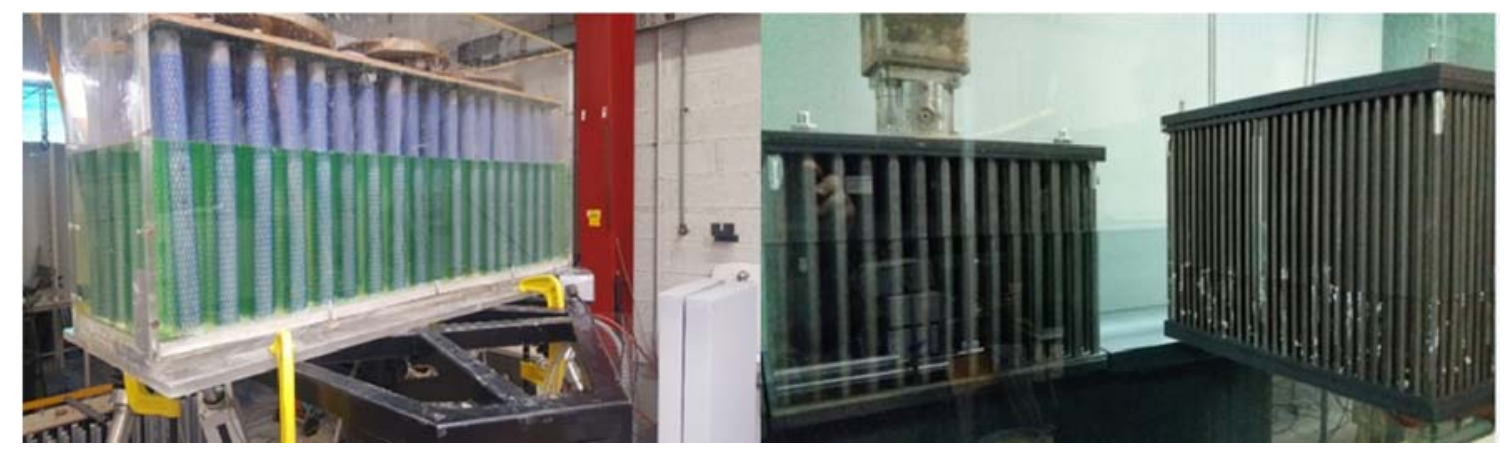

Figure 2. Illustration des expérimentations en hexapode et en canal.

Les méthodes et résultats de ces tests sont précisément décrits par BENOIT et al. (2020). De manière synthétique, les résultats montrent d'abord que le terme déterminant de la performance de dissipation est le coefficient de trainée, noté $\mathrm{C}_{\mathrm{d}}$, qui dépend non seulement des caractéristiques de l'ouvrage mais aussi des propriétés du milieu. Il a été observé qu'à porosité constante (la conservation de la porosité se traduit par une variation de la densité de pieux), l'atténuation des vagues augmente lorsque le diamètre des pieux diminue (et donc lorsque leur densité augmente). Les résultats ont confirmé le fait que lorsque la porosité diminue (i.e. quand le taux de remplissage par les pieux augmente), la performance d'atténuation augmente : pour une porosité variant de $90 \%$ à $70 \%$, les performances d'atténuation passent de $20 \%$ à $55 \%$. De plus, le déploiement d'un ouvrage à porosité décroissante de 90 à $70 \%$ permet d'atteindre des résultats similaires à ceux d'une porosité constante de $80 \%$ en termes d'atténuation de la hauteur des vagues (i.e. $45 \%$ de hauteur de vague), mais un tel ouvrage, à porosité décroissante, minimise la réflexion énergétique en façade de l'ouvrage ce qui limiterait le processus d'affouillement. Les tests ont montré qu'une augmentation de la rugosité de surface des cylindres conduit à une augmentation significative des performances. Ensuite, il est observé, pour de longs ouvrages, que l'essentiel de la dissipation se fait dans les premiers mètres. La quantité d'énergie de la houle dissipée diminue au fur et à mesure qu'elle se propage au travers du réseau de cylindre.

Ces premiers résultats nécessitent cependant d'être approfondis, notamment par rapport à la méthodologie du $\mathrm{Cd}$ et ne permettent pas encore d'aboutir directement à la conception d'un objet mais sont néanmoins utiles pour orienter les prochains travaux à réaliser. Ils seront utilisés dans la phase développement, et notamment de conception du module, pour définir les caractéristiques géométriques de la solution ROOT (les caractéristiques écologiques non hydrosédimentaires du module seront abordées par la suite dans la phase de développement) : 


\section{XVI'èmes Journées Nationales Génie Côtier - Génie Civil \\ Le Havre 2020}

- Sélection de pistes de travail appropriées en termes de performance. Typiquement, une réflexion peut être engagée sur la rugosité du matériau, et sa capacité à se concrétionner pour accroitre cette rugosité et donc sa performance.

- Définition de gammes de valeurs cibles. Il semble peu pertinent de réfléchir à un ouvrage excédant les $10 \mathrm{~m}$ de large, voire les $5 \mathrm{~m}$ étant donnés les objectifs de performance recherchés, et pour rester dans une solution réaliste d'un point de vue technico-économique. Des approches d'ouvrage à porosité variable de 90 à $70 \%$ semblent également pertinentes, en privilégiant des racines tortueuses de petits diamètres $(<12 \mathrm{~cm}$ a minima $)$.

- Essais et données complémentaires. Pour produire un outil d'aide à la décision adapté, des essais dans des configurations d'échelle moins réduite semblent pertinents à mener dans un objectif de calibration et d'intégration des dynamiques sédimentaires.

\section{Conclusion}

Le projet ROOT Recherche a pour objectifs d'évaluer la pertinence d'une solution d'atténuation de houle qui s'appuierait sur une géométrie inspirée par la mangrove, et d'en définir certaines propriétés clés (surface spécifique, tortuosité, porosité). Cette solution permettra de restaurer, sur un site dégradé, un contexte hydrosédimentaire approprié à sa recolonisation par la mangrove ou encore de maintenir et densifier un site en régression lorsque cette dernière est une conséquence de forçages hydrodynamiques trop élevés. La restauration de ces écosystèmes permettra alors, sur le long terme, le retour des services écosystémiques associés tels que, par exemple, le stockage du carbone ou encore la capacité à lutter contre les effets du changement climatique.

La pertinence de cette approche a été évaluée à partir de plusieurs tests conduits en laboratoire et en modèle numérique, sur la base de forêts de pieux exposées à différentes conditions de houle (BENOIT et al., 2020). De manière synthétique, les expérimentations conduisent aux conclusions clés suivantes :

- l'utilisation d'un ouvrage poreux constitué d'obstacles cylindriques verticaux, à l'image de racines émergentes, est pertinente du fait qu'on utilise relativement peu de matériaux (au contraire d'une digue basse par exemple) et que la mise en place reste peu couteuse.

- la porosité, la rugosité, la tortuosité, la largeur et la hauteur de l'ouvrage sont tous des paramètres influençant la performance d'atténuation au même titre que le diamètre des "racines" qui le constituent.

- pour une quantité de matériau équivalente, la rugosité de peau des pieux à une importance significative. Plus l'ouvrage est rugueux et plus il dissipe la houle.

- plus l'ouvrage est tortueux, plus il dissipe la houle.

- plus l'ouvrage est large (i.e. plus la distance parcourue par la houle au sein de l'ouvrage est longue) et plus il dissipe la houle. Cependant, la quantité d'énergie dissipée diminue 


\section{Thème 6 - Gestion durable des zones littorales et estuariennes}

au fur et à mesure que la houle se propage au travers de la solution et cet effet n'est pas linéaire.

- a porosité constante, plus le diamètre racinaire est faible (i.e. plus le nombre de racines augmente), plus l'ouvrage dissipe la houle. Ainsi, pour une quantité de matériau donnée, il faut privilégier le nombre de racines.

Ces premiers résultats permettent d'une part, de valider l'intérêt d'une structure poreuse pour dissiper de l'énergie de la houle et des courants et d'autre part, de poser un cadre aux réflexions prochaines concernant le design d'un objet ROOT et de l'ouvrage assemblé. Pour une porosité donnée, il semblerait que la perte de performance liée à l'optimisation budgétaire du diamètre racinaire (moins de racines, donc gain en termes de coûts de projet) puisse être compensée par une augmentation de la rugosité de surface. De plus, la possibilité d'atteindre des vitesses faibles à proximité du fond sédimentaire avec une emprise au sol inférieure à $20 \%$ permettrait de ménager à la fois la performance technique et la performance écologique par une meilleure oxygénation du substrat. Enfin, à l'échelle d'un ouvrage, et non d'un réseau racinaire, il est envisageable d'atteindre des performances élevées avec des ensembles lacunaires, tant que les caractéristiques de porosité et de tortuosité d'ensemble sont respectées, ce qui constituerait un argument majeur tant en termes de pertinence économique et technique que d'intégration paysagère. La phase de Recherche a ainsi permis (1) la sélection de plusieurs propriétés de l'ouvrage à approfondir dans le cadre du développement d'un ou plusieurs concepts, (2) la mise à l'écart de plusieurs propriétés qui n'auraient qu'une influence marginale sur la performance de l'ouvrage ou représentent des contraintes de mise en œuvre insurmontables à ce stade et (3) de s'assurer que certains modèles numériques existants, même s'ils ne représentent pas toute la complexité des phénomènes, permettent de tester certaines caractéristiques de l'ouvrage.

Dans la perspective de la mise au point d'une solution ROOT opérationnelle, la suite des travaux se focalisera sur le développement d'une solution qui répondra aux objectifs hydrosédimentaires, écologiques et sociétaux définis initialement sur le projet ROOT. Dans un premier temps, le chantier Conception permettra l'intégration les paramètres clefs qui ressortent de cette phase de recherche à un ou plusieurs designs de ROOT. Le volet hydrosédimentaire de ce chantier reposera sur les résultats de ROOT Recherche, sur l'intervention d'experts sectoriels (ateliers, entretiens), mais aussi sur des tests en modèle physique de la solution finale qui permettront la calibration et la validation d'un modèle numérique dont l'objectif sera de prévoir l'efficacité de la solution lors de son déploiement sur site. 


\section{XVİ̀mes Journées Nationales Génie Côtier - Génie Civil \\ Le Havre 2020}

\section{Références bibliographiques}

BALKE T., BOUMA T.J., HORSTMAN E. M., WEBB E.L., ERFTEMEIJER P.L., HERMAN P. M. (2011). Windows of opportunity: thresholds to mangrove seedling establishment on tidal flats. Marine Ecology Progress Series, Vol. 440, pp 1-9. https://doi.org/10.3354/meps09364

BENOIT M., BEUDIN A., DALLE J., KIMMOUN O., REMY F., WAELES B. (2020). Etudes expérimentales et numériques de la propagation des vagues à travers un réseau de cylindres verticaux en vue de la conception d'un système atténuateur de houle inspiré des racines de mangrove. JNGCGC 2020, Le Havre, (sous presse).

BLASCO F., AIZPURU M., GERS C., (2001). Depletion of the mangroves of continental Asia. Wetland Ecology Management, Vol. 9(3), pp 245-256. https://doi.org/10.1023/A:1011169025815

BULLERI F., CHAPMAN M.G. (2010). The introduction of coastal infrastructure as a driver of change in marine environments. Journal of Applied Ecology, Vol. 47(1), pp 26-35. https://doi.org/10.1111/j.1365-2664.2009.01751.x

CLOUGH B.F. (1992). Primary productivity and growth of mangrove forests. Tropical mangrove ecosystems. American Geophysical Union, Washington DC.

FAGHERAZZI S., BRYAN K., NARDIN W. (2017), Buried alive or washed away: the challenging life of mangroves in the Mekong delta, Oceanography, Vol. 30(3), pp 48-59. https://doi.org/10.5670/oceanog.2017.313

JONES C.G., LAWTON J.H., SHACHAK M. (1997). Positive and negative effects of organisms as physical ecosystem engineers. Ecology, Vol. 78(7), pp 1946-1957. https://doi.org/10.1890/0012-9658(1997)078[1946:PANEOO]2.0.CO;2

LEWIS III R.R. (2005). Ecological engineering for successful management and restoration of mangrove forests. Ecological engineering, Vol. 24(4), pp 403-418. https://doi.org/10.1016/j.ecoleng.2004.10.003

MARCHAND M. (2008), Mangrove restoration in Vietnam: Key considerations and practical guide, Deltares Report T2666, $42 \mathrm{p}$.

MAZDA Y., MAGI M., KOGO M., HONG P. N. (1997). Mangroves as a coastal protection from waves in the Tong King delta, Vietnam. Mangroves and Salt marshes, Vol. 1(2), pp 127-135. https://doi.org/10.1023/A:1009928003700

NARAYAN S., M. BECK B. REGUERO I. LOSADA B. VAN WESENBEECK N. PONTEE (2016), The effectiveness, costs and coastal protection benefits of natural and nature-based defences. PLoS ONE 11(5). https://doi.org/10.1371/journal.pone.0154735

SAENGER P., SIDDIQI N.A. (1993). Land from the sea: The mangrove afforestation program of Bangladesh. Ocean and Coastal Management, Vol. 20 (1), pp 23-39. https://doi.org/10.1016/0964-5691(93)90011-M

SCHEFFER M., CARPENTER S., FOLEY J.A., FOLKE C., WALKER B. (2001). Catastrophic shifts in ecosystems. Nature, Vol. 413(6856), 591. https://doi.org/10.1038/35098000 
Thème 6 - Gestion durable des zones littorales et estuariennes

SCHMITT K., ALBERS T., PHAM T., DINH S. (2013), Site-specific and integrated adaptation to climate change in the coastal mangrove zone of Soc Trang Province, Vietnam. Journal of Coastal Conservation, Vol. 17, pp 545-558. https://doi.org/10.1007/s11852$\underline{013-0253-4}$

VAN CUONG C., BROWN S., TO H.H., HOCKINGS M. (2015). Using Melaleuca fences as soft coastal engineering for mangrove restoration in Kien Giang, Vietnam. Ecological engineering, Vol. 81, pp 256-265. https://doi.org/10.1016/j.ecoleng.2015.04.031 ZHANG Q., WEN X., SONG C., LIU S. (1996). The measurement and study on sedimentation rates in mangrove tidal flats. Tropic Oceanology, Vol. 15(4), pp 57-62. 